\title{
Una estrategia de control mediante observadores para la temperatura en edificio de oficinas
}

\author{
Ruben Mascaró Palliser, Ramon Costa Castelló \\ Escola Tècnica Superior d'Eginyeria Industrial de Barcelona (ETSEIB)- UPC \\ rmascaropalliser@gmail.com,ramon.costa@upc.edu \\ José Domingo Álvarez Hervás \\ CIESOL - ceiA3. Dpto. de Informática, Universidad de Almería (UAL) \\ jhervas@ual.es
}

\section{Resumen}

En este trabajo se presenta un modelo térmico de una habitación basado en primeros principios junto con el modelo térmico de las paredes que la conforman. Ambos modelos permiten formular el modelo parametrizable de un edificio con independencia del número de habitaciones que tenga. Además, se presentan resultados utilizando el modelo de un sistema de control basado en realimentación de estados que ha sido diseñado tomando como referencia las técnicas de control LQR. El sistema de control utiliza un observador basado en un filtro de Kalman que permita estimar la temperatura de todas las paredes del edificio, de esta manera, una futura implementación del sistema de control en un edificio real permitiría un ahorro considerable en lo tocante a la red de sensores que éste necesitaría para su funcionamiento.

Palabras clave: Modelado de edificio; confort térmico; observadores

\section{Introducción}

En 2010 el sector de la construcción representó el $32 \%$ del consumo global de energía y un $19 \%$ del total de emisiones de $\mathrm{CO}_{2}$ expulsadas a la atmósfera [5]. A nivel europeo y de acuerdo con la directiva europea 2012/27/EU [1], a los estados miembros de la Unión Europea se les requiere suministrar una estrategia para la rehabilitación de edificios y, de esta manera, reducir la energía consumida por estos en un $20 \%$ para el año 2020 y así poder cumplir con el protocolo de Kyoto. No sólo se trata de una cuestión con un marcado impacto ecológico si no también financiero, el consumo de energía representa una cantidad importante de los gastos financieros si se tiene en cuenta todo el ciclo de vida del edificio [11].

Por todo lo anteriormente mencionado, la planificación y construcción de edificios energéticamente eficientes se han vuelto cuestiones cada vez más importantes para garantizar la sostenibilidad ecológica y económica. Desde la etapa más temprana del proceso de planificación del edificio se tienen que desarrollar análisis de costes y beneficios de la diversas alternativas que puden implementarse para aumentar el rendimiento energético de éste. Una manera de conocer el consumo energético de un edificio antes de su construcción es mediante el uso de modelos. De esta manera las medidas bioclimáticas que se quieran añadir al edificio pueden ser evaluadas antes de su implementación.

Por este motivo, la mayoría de los modelos disponibles que se pueden encontrar en la literatura están relacionados con la evaluación del rendimiento energético en edificios, como por ejemplo los trabajos presentados en [8, 9], aunque también se pueden encontrar modelos para el desarrollo de estrategias de control en edificios [4] o, incluso, trabajos que utilizan diferentes técnicas como redes neuronales (ANNs acronimo del inglés Artificial Neural Networks) para diseñar modelos de caja negra, black-box models [2]. En resumen, es posible encontrar una amplia variedad de modelos para propositos muy diferentes.

En este trabajo se presenta el modelo térmico de una habitación basado en primeros principios, que junto con el modelo térmico de las paredes permite formular el modelo de un edificio con independencia del número de habitaciones que tenga. El modelo destaca por su sencillez y por su parametrización lo que lo hace ideal para caracterizar un edificio independiente de su composición estructural. Dicho modelo puede ser utilizado posteriormente para el desarrollo de controladores de confort térmico como los presentados en [3, 6, 7, 10]. Como prueba de esto, en este trabajo se presentaran los resultados de un sistema de control basado en realimentación de estados que ha sido diseñado tomando como referencia las técnicas de control LQR. El sistema de control utiliza el modelo térmico de la habitación desarrollado en este trabajo junto con un observador basado en un filtro de Kalman que permita estimar la temperatura de todas las paredes del edificio. De esta manera, el coste de la red de sensores necesarios para implementar el sistema de control en un edificio real se vería drásticamente reducido aumentando la escalabilidad del controlador propuesto. 
El resto del trabajo se divide como sigue: la estructura del modelo es presentada en la sección 2, los parámetros del modelo utilizados en el ejemplo particular de este trabajo son listados en la sección 3 , la sección 4 es dedicada a un análisis de la respuesta del sistema autónomo mientras que el sistema de control propuesto es expuesto en la sección 5. Por último, los resultados del controlador propuesto así como las conclusiones son descritos en las secciones 6 y 7 , respectivamente.

\section{Modelo del edificio}

El modelo térmico utilizado en este trabajo se ha desarrollado tomando como referencia la disposición en planta de un edificio rectangular de nueve habitaciones también rectangulares, todas ellas iguales y dispuestas tal y como se muestra en la figura 1. Como hipótesis iniciales, se supone que cada habitación dispone de su propio sistema de climatización, el cual se asume como una fuente de excitación capaz de alterar la temperatura del aire dentro de la habitación. No se consideran, en principio, otras fuentes de excitación internas como pueden ser las personas, las luces, las ventanas, etc., aunque se prevé que puedan añadir-se fácilmente al modelo si se describen como perturbaciones. El objetivo es, de este modo, la creación de un modelo base suficientemente representativo de la dinámica del edificio para el ensayo de controladores, que pueda ser ampliado según las necesidades de cada estudio.

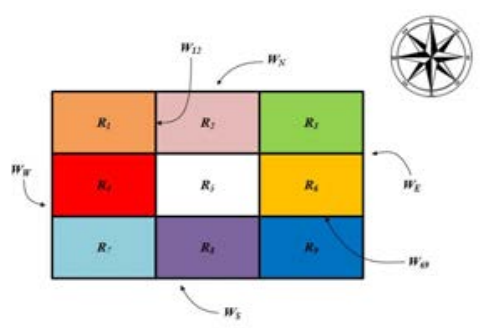

Figura 1: Representación geométrica de la planta del edificio

El modelo térmico del edificio, tal y como se concibe en este trabajo, puede ser descrito de forma relativamente sencilla si se piensa en el edificio como una malla gruesa en la que la excitación de la temperatura en una de las habitaciones provoca densidades de flujo de calor en las paredes de ésta. A modo de simplificación, se asume que no hay flujo de calor a través del suelo o el techo y que las temperaturas en las habitaciones y en las paredes son uniformes.

Partiendo de éstas hipótesis y de los principios básicos de transferencia de calor, el edificio se mo- dela como una malla de sistemas de primer orden donde los nodos representan las temperaturas en el interior de las habitaciones o en las paredes. Las ecuaciones del modelo son, de este modo, las relaciones entre los flujos de calor evaluados en cada uno de los nodos en forma de balances de energía.

Recordemos que cualquier ley de balance establece que la suma de la energía que entra en un contorno y la energía que se ha generado dentro es igual al incremento de energía interna. Así pues, podemos distinguir tres tipos de ecuaciones en el modelo según el tipo de nodo donde se realiza el balance:

- Ecuaciones de la dinámica del interior las habitaciones

- Ecuaciones de la dinámica de las paredes interiores

- Ecuaciones de la dinámica de las paredes exteriores

\subsection{Modelo dinámico del interior las habitaciones}

Cada habitación $R_{k}$ almacena una energía térmica $E_{k}^{R}[\mathrm{~J}]$ que es función de la temperatura a la que se encuentra el aire en su interior, $T_{k}^{R}[\mathrm{~K}]$. Esta relación puede aproximarse como:

$$
E_{k}^{R}=C_{k}^{R} T_{k}^{R}
$$

siendo $C_{k}^{R}[\mathrm{~J} / \mathrm{K}]$ la capacidad calorífica del aire contenido en la habitación. Así pues, la variación de energía o calor almacenado en dicha habitación es:

$$
Q_{k}^{R}=C_{k}^{R} \frac{d T_{k}^{R}}{d t} .
$$

Tal y como se ha planteado el modelo, esta variación de energía es debida al aporte de calor por medio del sistema de climatización de la habitación, $G_{k}^{R}[\mathrm{~W}]$ y al flujo de calor que entra por las cuatro paredes laterales, a las que llamaremos $W_{k, N}, W_{k, W}, W_{k, S}$ y $W_{k, E}$ según se encuentren en dirección norte, oeste, sur y este, respectivamente. De este modo, el balance de energía en el interior de la habitación $R_{k}$ queda:

$$
\begin{array}{r}
C_{k}^{R} \frac{d T_{k}^{R}}{d t}=G_{k}^{R}+K_{k}^{k, N}\left(T_{k, N}^{W}-T_{k}^{R}\right)+ \\
+K_{k}^{k, W}\left(T_{k, W}^{W}-T_{k}^{R}\right)+K_{k}^{k, S}\left(T_{k, S}^{W}-T_{k}^{R}\right)+ \\
+K_{k}^{k, E}\left(T_{k, E}^{W}-T_{k}^{R}\right)
\end{array}
$$

donde $K_{k}^{k, i}[\mathrm{~W} / \mathrm{K}]$ es la inversa de la resistencia térmica entre la pared $W_{k, i}$ y el aire de la habitación $R_{k}$ 


\subsection{Modelo dinámico de las paredes interiores}

Como ocurre con el aire contenido en las habitaciones, cada pared $W_{i, j}$ situada entre dos habitaciones $R_{i}$ y $R_{j}$ almacena una energía térmica, $E_{i, j}^{W}[\mathrm{~J}]$, que es función de su temperatura, $T_{i, j}^{W}[\mathrm{~K}]$. Esta relación se aproxima como:

$$
E_{i, j}^{W}=C_{i, j}^{W} T_{i, j}^{W}
$$

donde $C_{i, j}^{W}[\mathrm{~J} / \mathrm{K}]$ es la capacidad calorífica de la pared. La variación de energía almacenada en dicha pared resulta:

$$
Q_{i, j}^{W}=C_{i, j}^{W} \frac{d T_{i, j}^{W}}{d t}
$$

En este caso, la variación de energía, $Q_{i, j}^{W}$, en la pared $W_{i, j}$ es debida únicamente al flujo de calor procedente de sus habitaciones contiguas, $R_{i}$ y $R_{j}$, de modo que el balance queda:

$C_{i, j}^{W} \frac{d T_{i, j}^{W}}{d t}=-K_{i}^{i, j}\left(T_{i, j}^{W}-T_{i}^{R}\right)-K_{j}^{i, j}\left(T_{i, j}^{W}-T_{j}^{R}\right)$

\subsection{Modelo dinámico de las paredes exteriores}

Finalmente, se han de considerar las ecuaciones asociadas a las paredes exteriores del edificio. Dichas paredes se denotan como $W_{e}$, siendo el subíndice $e$ una letra indicativa de su orientación respecto los puntos cardinales. Así, $W_{N}$ será la pared norte, $W_{E}$ la pared este, $W_{S}$ la pared sur y $W_{W}$ la pared oeste.

Igual que las paredes interiores, cada pared exterior $W_{e}$ almacena una energía térmica $E_{e}^{W}[\mathrm{~J}]$ que depende linealmente de su temperatura $T_{e}^{W}[\mathrm{~K}]$ :

$$
E_{e}^{W}=C_{e}^{W} T_{e}^{W}
$$

siendo $C_{e}^{W}$ su capacidad calorífica.

La variación de energía almacenada en una pared exterior

$$
Q_{e}^{W}=C_{e}^{W} \frac{d T_{e}^{W}}{d t}
$$

depende del flujo de calor procedente del exterior y de las habitaciones $R_{k}$ con las que comunica y, en consecuencia, se expresa como:

$C_{e}^{W} \frac{d T_{e}^{W}}{d t}=-K_{\infty}^{e}\left(T_{e}^{W}-T_{\infty}\right)-\sum_{k \mid e \in k} K_{k}^{e}\left(T_{e}^{W}-T_{k}^{R}\right)$

donde $K_{\infty}^{e}[\mathrm{~W} / \mathrm{K}]$ es la inversa de la resistencia térmica entre la pared $W_{e} \mathrm{y}$ el exterior, $K_{k}^{e}[\mathrm{~W} / \mathrm{K}]$ la inversa de la resistencia térmica entre la pared $W_{e}$ y la habitación $R_{k}$ y $T_{\infty}$ la temperatura exterior.

\subsection{Modelo global}

Considérese ahora un edificio de $N \times M$ habitaciones. Tomando como variables de estado $\mathbf{x}$ las temperaturas de todos los nodos del modelo, como entradas $\mathbf{u}$ las potencias caloríficas suministradas por el equipo de climatización a las habitaciones y una perturbación $v$ que representa la temperatura exterior, el planteamiento de las ecuaciones anteriores para cada uno de los nodos lleva a la obtención de un sistema lineal de la forma:

$$
\dot{\mathbf{x}}=\mathbf{A} \mathbf{x}+\mathbf{B}_{\mathbf{u}} \mathbf{u}+\mathbf{B}_{\mathbf{v}} v
$$

siendo:

$$
\begin{aligned}
\mathbf{x} & :=\left[T_{1}^{R}, \ldots, T_{N \times M}^{R}, T_{1,2}^{W}, \ldots, T_{N \times M-1, N \times M}^{W}, \ldots,\right. \\
& \left.T_{n, \infty}^{W}, T_{w, \infty}^{W}, T_{s, \infty}^{W}, T_{e, \infty}^{W}\right]^{T} \in \mathbb{R}^{3(N \times M)+4-N-M} \\
\mathbf{u} & :=\left[G_{1}^{R}, G_{2}^{R}, \ldots, G_{N \times M}^{R}\right]^{T} \in \mathbb{R}^{N \times M} \\
v & :=T_{\infty} \in \mathbb{R} .
\end{aligned}
$$

En concreto, para el edificio de $3 \times 3$ habitaciones desarrollado en este trabajo, se tendrán 9 entradas (una por habitación) y 25 variables de estado. Además, suponiendo que únicamente son medibles las temperaturas del aire de las habitaciones,

$$
\mathbf{y}:=\left[T_{1}^{R}, T_{2}^{R}, \ldots, T_{9}^{R}\right]^{T}
$$

el modelo dinámico se describirá como:

$$
\begin{aligned}
\dot{\mathbf{x}} & =\mathbf{A} \mathbf{x}+\mathbf{B}_{\mathbf{u}} \mathbf{u}+\mathbf{B}_{\mathbf{v}} v \\
\mathbf{y} & =\mathbf{C} \mathbf{x}
\end{aligned}
$$

con $\mathbf{A} \in \mathbb{R}^{25 \times 25}, \mathbf{B}_{\mathbf{u}} \in \mathbf{R}^{25 \times 9}, \mathbf{B}_{\mathbf{v}} \in \mathbf{R}^{25 \times 1} \mathrm{y}$ $\mathbf{C} \in \mathbf{R}^{9 \times 25}$.

\section{Parámetros del modelo}

Los parámetros del modelo dinámico del edificio se han ajustado suponiendo que las habitaciones miden 5,53 $\mathrm{m}$ de largo, 4,96 $\mathrm{m}$ de ancho y $2,8 \mathrm{~m}$ de alto, lo que engloba un volumen de aire de $76,8 \mathrm{~m}^{3}$. Para las condiciones de temperatura a las que trabajará el modelo, se asume una densidad del aire de $1,175 \mathrm{~kg} / \mathrm{m}^{3}$ y un calor específico de $1006 \mathrm{~J} /(\mathrm{kg} \cdot \mathrm{K})$.

Por otra parte, se supone que las paredes tienen $20 \mathrm{~cm}$ de espesor y están hechas de bloques de hormigón convencionales. Para este material, se asume una densidad de $1250 \mathrm{~kg} / \mathrm{m}^{3}$, un calor específico de $920 \mathrm{~J} /(\mathrm{kg} \cdot \mathrm{K})$ y una conductividad térmica de $0,8 \mathrm{~W} /(\mathrm{m} \cdot \mathrm{K})$. El coeficiente de convección entre el aire y las paredes se ha establecido en $75 \mathrm{~W} /\left(\mathrm{m}^{2} \cdot \mathrm{K}\right)$.

A partir de estos datos es posible calcular todos los parámetros del modelo, los cuales se presentan resumidos en la Tabla 1. 


\begin{tabular}{|c|c|c|c|}
\hline Parámetro & Descripción & Valor & Unidades \\
\hline 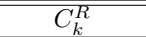 & Capacidad calorífica de la habitación $R_{k}$ & 90.751 & J/K \\
\hline$C_{i, j}^{W}(1)$ & Capacidad calorífica de la pared $W_{i, j}$ (lado largo) & 3.561 .320 & $\mathrm{~J} / \mathrm{K}$ \\
\hline$C_{i, j}^{W}(2)$ & Capacidad calorífica de la pared $W_{i, j}$ (lado corto) & 3.194 .240 & $\mathrm{~J} / \mathrm{K}$ \\
\hline$K_{i}^{i, j}(1)$ & Coeficiente de transferencia de calor entre la habitación $R_{i}$ y la pared $W_{i, j}$ (lado largo) & 111,93 & $\mathrm{~W} / \mathrm{K}$ \\
\hline$K_{i}^{i, j}(1)$ & Coeficiente de transferencia de calor entre la habitación $R_{i}$ y la pared $W_{i, j}$ (lado corto) & 100,40 & $\mathrm{~W} / \mathrm{K}$ \\
\hline$K_{\infty}^{k, \infty}(1)$ & Coeficiente de transferencia de calor entre el exterior y la pared $W_{k, \infty}$ (lado largo) & 335,80 & $\mathrm{~W} / \mathrm{K}$ \\
\hline$K_{\infty}^{k, \infty}(2)$ & Coeficiente de transferencia de calor entre el exterior y la pared $W_{k, \infty}$ (lado corto) & 301,19 & $\mathrm{~W} / \mathrm{K}$ \\
\hline
\end{tabular}

Tabla 1: Parámetros del modelo

\section{Evaluación de la respuesta del sistema autónomo}

El modelo del edificio se ha implementado en Simulink con el objetivo de poder ensayar distintas estrategias de control y estudiar sus prestaciones. Para verificar el correcto funcionamiento del modelo, se han llevado a cabo distintas simulaciones poniendo todas las entradas a cero $(\mathbf{u}=0)$ y haciendo que el sistema evolucione únicamente por acción de la temperatura exterior.

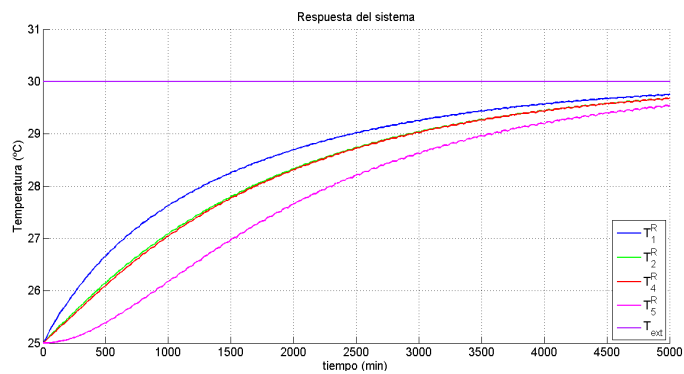

Figura 2: Respuesta del sistema autónomo

La figura 2 muestra algunos de los resultados obtenidos. En esta simulación se ha considerado que, inicialmente, la temperatura en el interior de todas las habitaciones (y la de las paredes) es de $25{ }^{\circ} \mathrm{C}$, mientras que la temperatura exterior es de $30^{\circ} \mathrm{C}$. Como se puede apreciar, las temperaturas de todas las habitaciones tienden a igualarse a la temperatura del exterior, situación en la que se alcanza el equilibrio térmico de todo el sistema. Sin embargo, el efecto aislante de las paredes hace que la evolución de las temperaturas en el interior del edificio sea muy lenta. Además, la simulación pone de manifiesto el hecho de que, cuanta más superficie de pared en contacto con el exterior tenga una habitación, más rápidamente evoluciona la temperatura en su interior (véase que la temperatura de la habitación 1, situada en la esquina noroeste del edificio, crece más rápidamente que la de la habitación 5 , situada en el interior del mismo).

\section{Estrategia de control}

Con el fin de regular las temperaturas en el interior de las distintas habitaciones, se propone un sistema de control basado en realimentación de estado que ha sido diseñado tomando como referencia las técnicas de control LQR. Para ello, se ha impuesto que la ley de control $\mathbf{u}=-\mathbf{K} \mathbf{x}$ minimice una función de coste cuadrática del tipo

$$
J(u)=\int_{0}^{\infty}\left(\mathbf{x}^{T} \mathbf{Q} \mathbf{x}+\mathbf{u}^{T} \mathbf{R u}\right) d t
$$

sujeta a la dinámica del sistema

$$
\dot{\mathbf{x}}=\mathbf{A x}+\mathbf{B}_{\mathbf{u}} \mathbf{u} \text {. }
$$

En el caso de este trabajo, se asume que únicamente se tendrán medidas de la temperatura del aire en el interior de las habitaciones. Por este motivo, se ha optado por incorporar un observador basado en un filtro de Kalman que permita estimar la temperatura de todas las paredes del edificio. Además, con el objetivo de poder seguir la referencia, se ha diseñado un módulo de precompensación $\mathbf{K}^{*}$ que hace que el sistema global tenga ganancia unitaria.

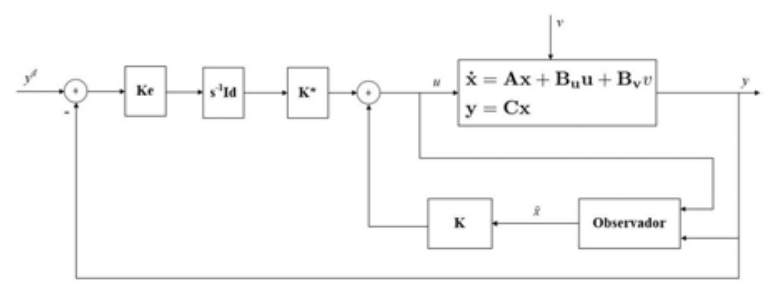

Figura 3: Esquema del sistema de control planteado

Finalmente, como el conjunto de sistemas resultante es de tipo 0 , se ha decidido incrementar el tipo del sistema añadiendo un conjunto de integradores en el controlador, obteniéndose la estructura de control de la figura 3 .

\section{Resultados de la simulación}

El sistema de control diseñado en este trabajo se ha implementado junto con el modelo del edificio 
previamente desarrollado en Simulink. En las simulaciones que aquí se presentan, se ha supuesto que inicialmente todas las habitaciones se encuentran a una temperatura de $28^{\circ} \mathrm{C}$, igual que todas las paredes del edificio. A continuación se detallan los resultados obtenidos.

Para la ejecución de la primera simulación, se ha programado una consigna de $24{ }^{\circ} \mathrm{C}$ en todas las habitaciones, suponiendo una temperatura exterior constante de $30^{\circ} \mathrm{C}$. En la figura 4 se representa la evolución de la temperatura en el interior de cuatro habitaciones distintas.

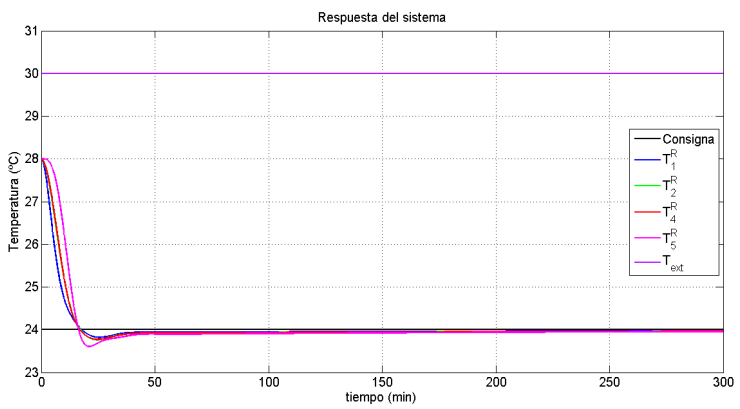

Figura 4: Resultados de la simulación para consignas iguales en todas las habitaciones y temperatura exterior constante

Como se puede ver, el sistema de control es capaz de llevar la temperatura de todas las habitaciones hacia el valor deseado. Si bien es cierto que la respuesta es relativamente lenta, se comprueba que a partir de los 14 minutos la temperatura de todas las habitaciones sufre una variación inferior a $0,5{ }^{\circ} \mathrm{C}$ respecto a la temperatura programada. La evolución de las temperaturas presenta un leve sobrepico, cuya desviación máxima respecto a la temperatura programada se da en la habitación 5 y es de $0,4{ }^{\circ} \mathrm{C}$. A los 35 minutos, una vez superado este sobrepico, el error ya es inferior a $0,2{ }^{\circ} \mathrm{C}$ en todas las habitaciones y empieza a decrecer de forma monótona, tendiendo lentamente a cero.

El hecho de tener un pequeño error que persiste durante un tiempo considerable se considera aceptable dada la complejidad del sistema a controlar. De hecho, puede conseguirse que el error tienda a cero en un tiempo menor si se aumenta el parámetro $Q$ del controlador o bien la ganancia proporcional $K_{e}$ que multiplica a los integradores. Sin embargo, al incrementar el valor de estos parámetros aumentan notoriamente las oscilaciones en el transitorio, lo cual no interesa en este tipo de sistema. Por tanto, debe establecerse un compromiso entre el interés por conseguir que la respuesta tienda de forma rápida al valor de la consigna y el de intentar evitar oscilaciones bruscas en el transitorio.
Una vez estudiado el comportamiento del sistema para consignas iguales y temperatura exterior constante, se ha realizado una segunda simulación en la que se evalúa el comportamiento del sistema ante una perturbación (variación de la temperatura exterior). Los resultados se presentan en la figura 5 .

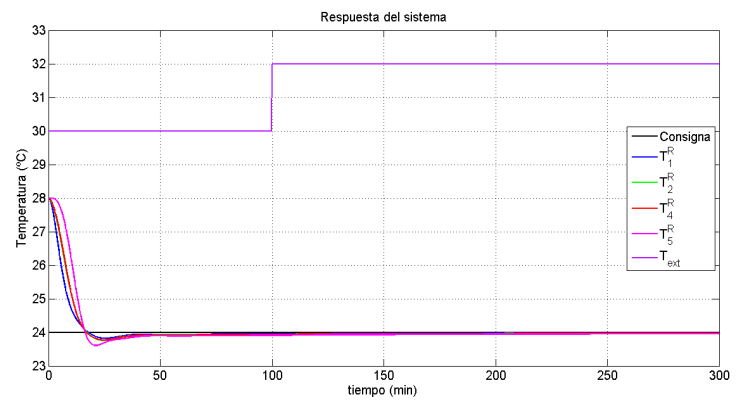

Figura 5: Resultados de la simulación para consignas iguales en todas las habitaciones y temperatura exterior variable

En este caso se observa que la variación en la temperatura exterior no altera prácticamente la respuesta del sistema. El motivo es que el sistema responde muy lentamente a cambios en la temperatura exterior debido al efecto aislante de las paredes, como ya se ha visto en la sección 4. De este modo, el sistema de control es capaz de compensar el cambio de temperatura exterior antes de que éste afecte apreciablemente a las temperaturas del interior del edificio.

Finalmente, vista la capacidad del controlador de compensar cambios en la temperatura exterior, se han realizado varias simulaciones programando distintas consignas de temperatura en cada una de las habitaciones. Las temperaturas programadas se detallan en la Tabla 2, mientras que en la figura 6 se muestran los resultados de la simulación. Véase que, en este caso, se ha supuesto una temperatura exterior constante de $30^{\circ} \mathrm{C}$.

\begin{tabular}{|r|c|c|c|c|c|c|c|c|c|}
\hline Habitación & 1 & 2 & 3 & 4 & 5 & 6 & 7 & 8 & 9 \\
\hline Consigna $\left({ }^{\circ} \mathbf{C}\right)$ & 25 & 22 & 24 & 24 & 23 & 22 & 21 & 26 & 24 \\
\hline
\end{tabular}

Tabla 2: Consignas de temperatura

Los resultados ponen de manifiesto la capacidad del sistema de control de llevar la temperatura de cada habitación al valor deseado, independientemente de cuál sea la temperatura en las habitaciones contiguas. Se observa además que, a los 15 minutos, la temperatura en todas las habitaciones difiere en menos de $0,5{ }^{\circ} \mathrm{C}$ del valor programado. Igual que en las simulaciones anteriores, la evolución de las temperaturas presenta un leve sobrepico que en ningún caso supera los $0,45^{\circ} \mathrm{C}$ respecto a 


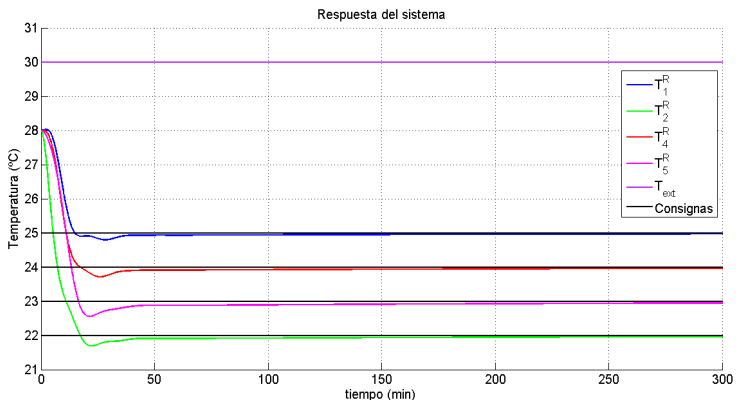

Figura 6: Resultados de la simulación para consignas distintas en las habitaciones y temperatura exterior constante

la temperatura programada, y tras el cual el error tiende de forma monótona a cero.

También se puede observar en este caso que un cambio en la temperatura exterior no afecta de forma perceptible la evolución de las temperaturas en el interior del edificio, como muestra la figura 7

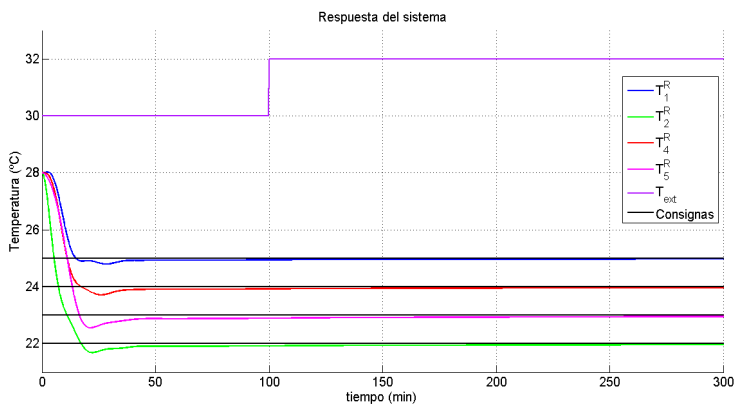

Figura 7: Resultados de la simulación para consignas distintas en las habitaciones y temperatura exterior variable

\section{Conclusiones}

En este trabajo se ha presentado el modelo térmico de una habitación basado en primeros principios junto con los modelos del entorno que la conforman (paredes, techo y suelo). Estos dos modelos parametrizables permiten formular el modelo global de un edificio con independencia del número de habitaciones y de la estructura que tenga.

Como prueba de la utilización del modelo se ha desarrollado, basado en éste, un sistema de control basado en realimentación de estados que ha sido diseñado tomando como referencia las técnicas de control LQR. El sistema de control, que presenta buenos resultados en simulación realizando un seguimiento de consigna de temperatura, utiliza un observador basado en un filtro de Kalman que permita estimar la temperatura de todas las paredes del edificio. De esta manera, el coste de la red de sensores necesarios para implementar el sistema de control en un edificio real se vería drásticamente reducido aumentando la escalabilidad del controlador propuesto.

Como trabajo futuro se va a intentar la implantación del sistema de control en un edificio para obtener resultados reales que certifiquen los expuestos en este trabajo.

\section{Agradecimientos}

Este trabajado ha sido parcialmente financiado con las becas de colaboración del MEC, los proyectos DPI2015-69286-C3-2-R, DPI2014-56364-C2-1R (MINECO/FEDER) y el proyecto 2014 SGR 267 de la AGAUR (agencia de la Generalitat de Catalunya). José Domingo Álvarez Hervás pertenece al programa del MEC 'Ramón y Cajal' cofinanciado por la fundación social Europea.

\section{Referencias}

[1] 2012/27/UE. Directive 2012/27/ue of the european parliament and of the council of $25 \mathrm{oc}-$ tober 2012 on energy efficency. disponible en linea en: link. último accesso 31 Mayo 2016. 2012.

[2] M. Castilla, J.D. Álvarez, M.G. Ortega y M.R. Arahal. Neural network and polynomial approximated thermal comfort models for hvac systems. Building and Environment, 59:107-115, 2013.

[3] A. I. Dounis y C. Caraiscos. Advanced control systems engineering for energy and comfort management in a building environment - a review. Renewable and Sustainable Energy Reviews, 13:1246-1261, 2009.

[4] I. Hazyuk, C. Ghiaus y D. Penhouet. Optimal temperature control of intermittently heated buildings using model predictive control: Part i-building modeling. Building and Environment, 51:379-387, 2012.

[5] O. Lucon, D. Ürge-Vorsatz, A. Zain Ahmed, H. Akbari, P. Bertoldi, L. F. Cabeza, N. Eyre, A. Gadgil, L. D. D. Harvey, Y. Jiang et al. Buildings. Climate change, 2014.

[6] P. D. Moroşan, R. Bourdais, D. Dumur y J. Buisson. A distributed MPC strategy based on benders' decomposition applied to multi-source multi-zone temperature regulation. Journal of Process Control, 21(5):729$737,2011$.

[7] F. Oldewurtel, A. Parisio, C. N. Jones, M. Morari, D. Gyalistras, M. Gwerder, 
V. Stauch, B. Lehmann y K. Wirth. Energy efficient buildings climate control using stochastic model predictive control and weather predictions. En American Control Conference (ACC10), Baltimore, Maryland, USA. 2010.

[8] T. Olofsson y T. M. I. Mahlia. Modeling and simulation of the energy use in an occupied residential building in cold climate. Applied Energy, 91(1):432-438, 2012.

[9] D. Saelens, W. Parys y R. Baetens. Energy and comfort performance of thermally activated building systems including occu- pant behavior. Building and Environment, 46(4):835-848, 2011.

[10] H. F. Scherer, M. Pasamontes, J. L. Guzmán, J. D. Álvarez, E. Camponogara y J. E. Normey-Rico. Efficient building energy management using distributed model predictive control. Journal of Process Control, 24(6):740-749, 2014.

[11] C. Stoy, S. Pollalis y D. Fiala. Estimating buildings' energy consumption and energy costs in early project phases. Facilities, 27(5/6):187-201, 2009. 